\title{
Using Personal Devices to Facilitate Multi-user Interaction with Large Display Walls
}

\author{
Ulrich von Zadow \\ Interactive Media Lab \\ Technische Universität Dresden \\ Dresden, Germany \\ uzadow@acm.org
}

\begin{abstract}
Large display walls and personal devices such as Smartphones have complementary characteristics. While large displays are well-suited to multi-user interaction (potentially with complex data), they are inherently public and generally cannot present an interface adapted to the individual user. However, effective multi-user interaction in many cases depends on the ability to tailor the interface, to interact without interfering with others, and to access and possibly share private data. The combination with personal devices facilitates exactly this. Multi-device interaction concepts enable data transfer and include moving parts of UIs to the personal device. In addition, hand-held devices can be used to present personal views to the user. Our work will focus on using personal devices for true multi-user interaction with interactive display walls. It will cover appropriate interaction techniques as well as the technical foundation and will be validated with corresponding application cases.
\end{abstract}

\section{Author Keywords}

Cross-device interaction; data transfer; mobile phones; display wall; wearable display; multi-user; collaboration

\section{ACM Classification Keywords}

H.5.2. Information Interfaces and Presentation: User Interfaces: Input devices and strategies, Interaction styles

\section{INTRODUCTION}

Wall-sized interactive displays are becoming more common and have been shown to provide numerous benefits [2]. Their potentially very high resolution means that they are usable from a distance as well as in reach of the hands, making them suitable for the exploration of large amounts of data. Collaboration is well-supported and physical navigation - moving around to access data - becomes possible, exploiting human spatial awareness [4]. At the same time, they have inherent

Permission to make digital or hard copies of part or all of this work for personal or classroom use is granted without fee provided that copies are not made or distributed for profit or commercial advantage and that copies bear this notice and the full citation on the first page. Copyrights for third-party components of this work must be honored. For all other uses, contact the Owner/Author. Copyright is held by the owner/author(s). UIST '15 Adjunct, November 08-11, 2015, Charlotte, NC, USA. ACM 978-1-4503-3780-9/15/11.

http://dx.doi.org/10.1145/2815585.2815592 limitations. Data shown is generally visible by all collaborators and thus public. It is hard to identify the user and provide a user-specific interface. In addition, it is unclear where user interface elements such as tool palettes should be placed on a very large display [2].

Contrast this with devices such as smartphones and tablets: They are inherently personal devices that connect users with their digital ID, providing easy access to private data in everyday situations. Due to their small size, personal devices have limited ability to show large amounts of data; also, sharing views with other people is hard at best. The combination of both device classes makes it possible to use the modalities - display output, touch input, sound and haptic feedback - available on the personal device to extend interaction with the large display. It also allows personalized interaction and access to private data when using the large display: User interface components such as palettes can be offloaded to the personal device, and data can be transferred in both directions, essentially moving it from private to public space and back (e.g., [13]).

\section{PREVIOUS WORK}

Interaction with large displays and personal mobile devices is an active research field. We first discuss work that examines relevant aspects of interactions with large vertical displays. The second section focuses on interaction with large displays using personal devices.

\section{Interaction with Wall Displays}

For a general introduction to interaction with high-resolution wall-sized displays, we refer to two overviews. Ni et al. [10] provide a comprehensive survey that covers rendering technology, interaction techniques and application domains. Among others, they mention transitioning between working within touching distance and further away as interaction challenge. Andrews et al. [2] give an overview of data visualization on large displays. Like other work of this group (e.g., [3, $4]$ ), this work asserts that physical navigation (moving around in front of the wall to access content) has advantages in speed and maintaining context. They emphasize that the "design of other interactions must afford or even exploit physical navigation" and argue that interaction should be localized: The user's position and focus should determine the location of interaction effects. For a different perspective on the effects of physical navigation, see [9]. Related to this is Greenberg et 

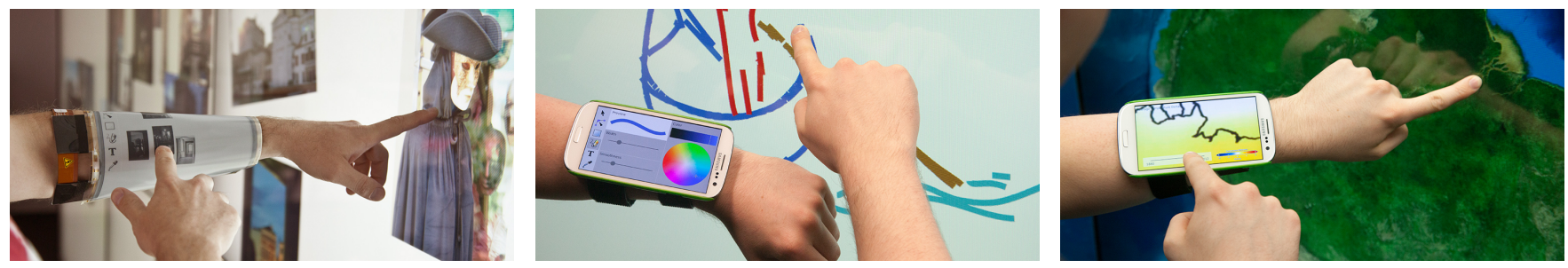

Figure 1. SleeD: e-Ink mockup (left), smartphone-based prototype with tool palette (center) and interactive personal view using the non-dominant SleeD hand to provide a frame of reference (right).

al.'s notion of Proxemic Interaction (e.g., [7]), in which interactions are based on spatial relationships between people and devices.

\section{Multi-Device Interaction with Large Displays}

There is a large body of work that covers interaction at close distance between personal devices and large displays. For space reasons, we restrict ourselves to a selection that covers a wide range of interaction techniques. Alt et al. [1] analyse content exchange techniques with public displays, comparing direct interaction with remote website and mobile phone interaction. Schmidt et al. [13] examined cross-device interaction between phones and interactive surfaces, building on the ability to detect touches by phones on a tabletop. They proposed a collection of interaction techniques in areas such as data transfer, personalization, or localized feedback. Using a mobile device to personalize interfaces was also investigated by Spindler et al. [15], who offload user interface palettes onto secondary hand-held displays and thereby free the associated display space. The idea of using mobile personal palettes is also found in Haller et al.'s work on the NiCE Discussion Room (e.g., [8]), who use pen interaction with static, palm-sized printed menus to interface with a large interactive whiteboard.

Interaction from a distance has also been examined, among others by Dachselt and Buchholz [6], who use expressive phone gestures for data transfer and interaction with large screen content. With CodeSpace, Bragdon et al. [5] integrate multi-device interaction and distal pointing into a realistic application scenario. In PointerPhone [14], Seifert et al. investigate the interactions possible when remote pointing is combined with interactions on the phone. Significantly, Peck et al.'s work [11] is to our knowledge the only one that combines pointing and physical navigation. In general, only few works propose interaction techniques that exploit the advantages of physical navigation. Finally, several authors (among them Rashid et al. [12]) investigate the effects of attention switches between small devices and large displays and find them time-consuming and disrupting. Interaction techniques that explicitly address this issue have not been investigated.

\section{RESEARCH OBJECTIVES}

The main goal of our work will be to assess the implications of using personal devices in the context of multi-user interaction with large display walls. A necessary prerequisite for this (and a research goal in itself) is enabling the association of touches on the wall to individual users. A further central objective is the development of appropriate interaction techniques. In this context, the following issues need to be taken into account:

- We agree with Andrews et al. [2] that techniques should adjust to a user's changing physical location and work handin-hand with physical navigation. As a user moves from overview distance to a close detail view, the enabled interactions need to follow perception in scale and precision as well as adjust to the user's level of engagement [7].

- Gaze switches are an inevitable part of multi-device interaction but have been shown to disrupt users and slow them down (e.g., [12]). They therefore need to be considered explicitly when designing interactions.

- Interactions need to enable smooth transitions between working directly with the wall (using, e.g., touch) and interaction at a distance (using the personal device or gestures) [10].

The work above will enable us to analyze the tradeoffs involved in different device configurations: What benefits and drawbacks do different types of personal devices have when compared to direct interaction with the display wall? A number of tradeoffs are involved here: While personal devices make working with private data easy, provide an implicit user identification, and afford interaction at a distance without changing modalities, directly interacting with the wall avoids gaze switches entirely and enables larger personal views. Our work should enable us to provide a well-founded analysis of these tradeoffs as well as associated recommendations for application designers.

\section{WORK TO DATE}

Work until now has focused on the one hand on development of a solid technical foundation. On the other hand, we have explored interaction between arm-worn devices and large display walls in the SleeD project [16].

As a technical foundation, we wanted a framework that allows rapid prototyping of novel user interfaces and offers support for seamless multi-device development. Furthermore, we required access to all needed input modalities: touch and position information for all connected devices. To fulfill these requirements, we extended the libavg framework ${ }^{1}$. Significant work went into supporting development of distributed user interfaces. The UI streaming component we wrote renders all user interfaces on a central server and streams the display contents via h264 over RTP to attached mobile devices.

\footnotetext{
${ }^{1}$ https://www.libavg.de
} 

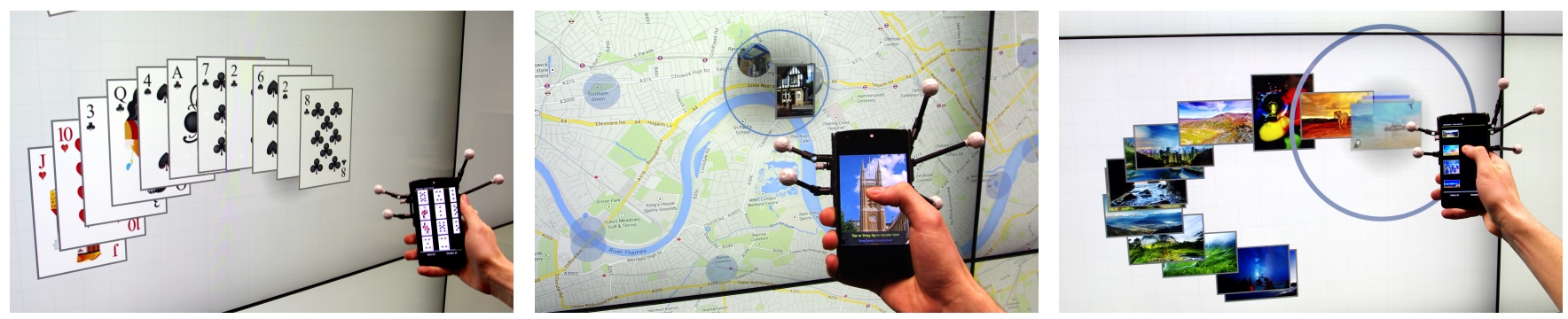

Figure 2. Exploring cross-device data transfer: Integrating layout support (left), transfer based on geolocation (center), and transfer of continuous streams of items (right).

Conversely, input events are streamed to the server. To the application developer, the different devices appear as local windows; all networking is abstracted away entirely. These components have been deployed successfully. As a result, libavg is being used as basis for nearly all wall and multidevice research at our lab.

With SleeD [16], we presented a concept for combining interactions on touch-sensitive sleeve displays (the SleeD) with large display walls. In wall interaction, a SleeD inherits the advantages (such as complex personalized interaction and access to private data) that other personal devices have. Additionally, it is quicker in activation and makes seamless switching to bimanual work on the wall possible. In our publication, we discuss different levels of coupling between wearable and wall. A focus is on close physical coupling, where the SleeD hand provides a frame of reference for the dominant hand (Figure 1, right). In addition, we propose novel user interface techniques that support data transfer, user-specific interfaces, and arbitrary personal views (Figure 1) that we verified using a qualitative study.

SleeD is a first major step in tackling the overall research objectives and lays the foundation for comparing use of personal devices with direct wall interaction. However, while it supports multi-user interaction conceptually, a multi-user capable implementation is still missing since the wall does not distinguish between users.

\section{CURRENT AND FUTURE WORK}

Current and future work will focus on three areas: the technical foundation that enables the remainder of the research, development and assessment of interaction techniques, and applications that validate the techniques.

\section{Technical Foundation}

Work on the technical foundation is for the most part done. However, one unsolved issue remains: How do we correlate users with touches at a display wall? Without this capability, personalized multi-user touch interaction is not possible. Numerous solutions for touch user identification have been proposed for tabletop displays, but most of these do not generalize to wall displays because they are either specific to the orientation, rely on viewing the touch from above or are limited to small displays.

We are developing a practically usable technical solution that serves as a basis for our development of application use cases, besides being a novel contribution in itself. This precludes development of custom hardware. We aim to have robust user detection and thus temporary user IDs for the duration of a typical usage session. This is complemented with stateof-the-art login procedures when a user enters the session to correlate persistent user IDs with the temporary IDs.

\section{Interaction Techniques}

We are currently investigating bidirectional data transfer techniques between smartphone and display wall (Figure 2) that make use of the phone's position and orientation. The techniques we are developing support multi-item transfer and layout, allow casual as well as precise interaction, work well with physical navigation, and are designed to minimize gaze switches between the devices. Our work includes a comprehensive qualitative study as well as an analysis of the underlying design space.

A second - related - project is a pointing technique that adapts to varying distances and thus works well with physical navigation. By seamlessly varying the pointing parameters, we can support casual and imprecise pointing at a distance and very precise pointing when close to the wall, while maintaining an imperceptible transition between the two.

Furthermore, we intend to use the work on SleeD as a basis for a comparison of using different modalities (smartphones, direct wall interaction and different arm-worn devices) for interaction with a display wall. We will build state-of-the-art interfaces for these modalities that support handling of personal data and data transfer, tool palettes, and individualized views on data and use these interfaces to conduct a comprehensive qualitative study. Our goal is to analyze the tradeoffs: How do the differences in the modalities with respect to private data handling, disruption through gaze switches, bimanual interaction on the wall, and user identification affect users?

\section{Applications}

In this part of the work, we will build application cases that validate the concepts and interaction techniques presented above. Currently, two application cases are planned: A game and a biological visualization application. The first is a timecritical cooperative multiplayer game (Figure 3, left) that we will use to evaluate behavior when using a variety of input modalities. Interesting questions include communication channels, awareness of other player's actions, and modes of cooperation.

The second application case involves visualization of biological data (Figure 3, right). In cooperation with biologists from 

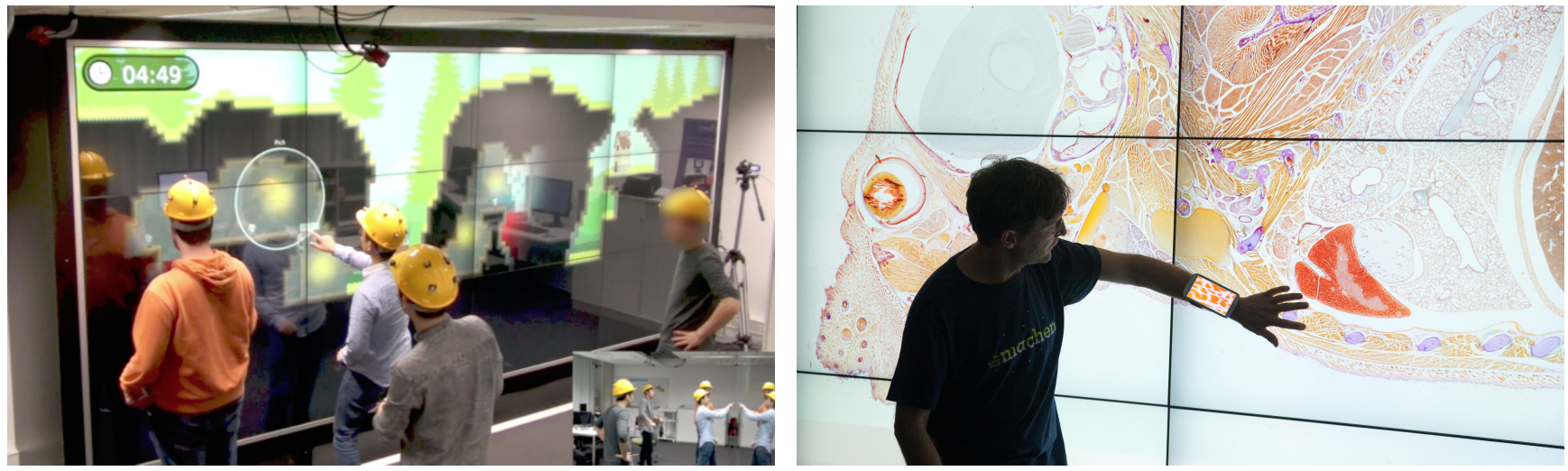

Figure 3. Application cases: Cooperative game (left) and focus-context interaction using biological data.

the Max Plank Institute for Molecular Cell Biology and Genetics, we plan to build a visualization application for light sheet microscopy images. Development will proceed in close cooperation with prospective users. The work on interaction techniques described above should on the one hand be a solid foundation for this work. On the other hand, we can use the application case to verify their practical usefulness.

\section{CONCLUSION}

Personal devices have the potential to enable true multi-user interaction with large display walls, and we have identified a number of research questions in this area that remain unsolved. We are confident that we can make a significant contribution in this area with the combination of technical foundation, interaction techniques, and verification using application cases.

\section{ACKNOWLEDGEMENTS}

I thank my advisor, Raimund Dachselt, for his indispensable help and guidance. Furthermore, I thank Ulrike Kister and Ricardo Langner for their significant assistance with the camera ready version.

\section{REFERENCES}

1. Alt, F., Shirazi, A. S., Kubitza, T., and Schmidt, A. Interaction techniques for creating and exchanging content with public displays. In Proc. $\mathrm{CHI}$, ACM (2013), 1709-1718.

2. Andrews, C., Endert, A., Yost, B., and North, C. Information visualization on large, high-resolution displays: Issues, challenges, and opportunities. Information Visualization 10, 4 (2011), 341-355.

3. Andrews, C., and North, C. The impact of physical navigation on spatial organization for sensemaking. IEEE Transactions on Visualization and Computer Graphics 19, 12 (2013), 2207-2216.

4. Ball, R., North, C., and Bowman, D. A. Move to improve: promoting physical navigation to increase user performance with large displays. In Proc. $\mathrm{CHI}$, ACM (2007), 191-200.

5. Bragdon, A., DeLine, R., Hinckley, K., and Morris, M. R. Code space: Combining touch, devices, and skeletal tracking to support developer meetings. In Proc. ITS, ACM (2011), 212-221.
6. Dachselt, R., and Buchholz, R. Natural throw and tilt interaction between mobile phones and distant displays. In Proc. CHI EA, ACM (2009), 3253-3258.

7. Greenberg, S., Marquardt, N., Ballendat, T., Diaz-Marino, R., and Wang, M. Proxemic interactions: the new ubicomp? Interactions 18, 1 (Jan. 2011), 42-50.

8. Haller, M., Leitner, J., Seifried, T., Wallace, J. R., Scott, S. D., Richter, C., Brandl, P., Gokcezade, A., and Hunter, S. The NiCE discussion room: Integrating paper and digital media to support co-located group meetings. In Proc. CHI, ACM (2010), 609-618.

9. Jakobsen, M. R., and Hornbæk, K. Is moving improving?: Some effects of locomotion in wall-display interaction. In Proc. CHI, ACM (2015), 4169-4178.

10. Ni, T., Schmidt, G., Staadt, O., Livingston, M., Ball, R., and May, R. A survey of large high-resolution display technologies, techniques, and applications. In Virtual Reality Conference, 2006 (march 2006), 223 - 236.

11. Peck, S. M., North, C., and Bowman, D. A multiscale interaction technique for large, high-resolution displays. In Proc. 3DUI, ACM (2009), 31-38.

12. Rashid, U., Nacenta, M. A., and Quigley, A. The cost of display switching: a comparison of mobile, large display and hybrid ui configurations. In Proc. AVI, ACM (2012), 99-106.

13. Schmidt, D., Seifert, J., Rukzio, E., and Gellersen, H. A cross-device interaction style for mobiles and surfaces. In Proc. DIS, ACM (2012), 318-327.

14. Seifert, J., Bayer, A., and Rukzio, E. PointerPhone: Using mobile phones for direct pointing interactions with remote displays. In Proc. INTERACT. Springer, 2013, 18-35.

15. Spindler, M., Cheung, V., and Dachselt, R. Dynamic tangible user interface palettes. In Proc. INTERACT. Springer, 2013, 159-176.

16. von Zadow, U., Büschel, W., Langner, R., and Dachselt, R. Sleed: Using a sleeve display to interact with touch-sensitive display walls. In Proc. ITS, ACM (2014), 129-138. 